OPEN ACCESS

Edited by:

Milica S. Prostran,

University of Belgrade, Serbia

Reviewed by:

Alberto Porta,

Università degli Studi di Milano, Italy Antonio Ivano Triggiani,

University of Foggia, Italy

*Correspondence:

Jiun-Chi Huang

karajan77@gmail.com

Received: 10 December 2017 Accepted: 06 February 2018

Published: 20 February 2018

Citation:

Niu S-W, Huang J-C, Chen S-C,

Lin HY-H, Kuo I-C, Wu P-Y,

Chiu Y-W and Chang J-M (2018)

Association between Age and Changes in Heart Rate Variability

after Hemodialysis in Patients with

Diabetes.

Front. Aging Neurosci. 10:43. doi: 10.3389/fnagi.2018.00043

\section{Association between Age and Changes in Heart Rate Variability after Hemodialysis in Patients with Diabetes}

\author{
Sheng-Wen Niu1,2,3, Jiun-Chi Huang1,3,4,5*, Szu-Chia Chen 1,3,4,5, Hugo Y.-H. Lin 1,2,5,6, \\ I.-Ching Kuo 1,2,3, Pei-Yu Wu ${ }^{3,4}$, Yi-Wen Chiu ${ }^{1,5}$ and Jer-Ming Chang ${ }^{1,5,7}$
}

1 Division of Nephrology, Department of Internal Medicine, Kaohsiung Medical University Hospital, Kaohsiung Medical University, Kaohsiung, Taiwan, ${ }^{2}$ Department of Internal Medicine, Kaohsiung Municipal Ta-Tung Hospital, Kaohsiung Medical University Hospital, Kaohsiung Medical University, Kaohsiung, Taiwan, ${ }^{3}$ Graduate Institute of Clinical Medicine, College of Medicine, Kaohsiung Medical University, Kaohsiung, Taiwan, ${ }^{4}$ Department of Internal Medicine, Kaohsiung Municipal Hsiao-Kang Hospital, Kaohsiung Medical University, Kaohsiung, Taiwan, ${ }^{5}$ Faculty of Medicine, College of Medicine, Kaohsiung Medical University, Kaohsiung, Taiwan, ${ }^{6}$ Graduate Institute of Medicine, College of Medicine, Kaohsiung Medical University, Kaohsiung, Taiwan, ${ }^{7}$ Department of Internal Medicine, Kaohsiung Municipal Cijin Hospital, Kaohsiung Medical University, Kaohsiung, Taiwan

Background: Heart rate variability (HRV) represents changes in the time between successive heart beats, and it has been used to assess the autonomic nervous system. Previous studies have reported autonomic dysfunction in diabetic patients undergoing hemodialysis (HD), however, no studies have evaluated the effects of age on changes in HRV in these patients. The aim of this study was to examine the effects of age on changes in HRV in diabetic HD patients.

Methods: We enrolled 84 diabetic patients receiving maintenance HD. HRV was measured before and after HD to assess changes in HRV $(\Delta \mathrm{HRV})$. The patients were divided into two groups based on their age (65 years $<$ or $\geq 65$ years).

Results: Compared to the patients aged $<65$ years, those aged $\geq 65$ years had a higher high frequency (HF) \% $(p=0.032)$ before HD. The patients aged $<65$ years had a significant increase in very low frequency, low frequency (LF), and HF after HD. The patients aged $\geq 65$ years had a significant increase in LF, but a significant decrease in $\mathrm{HF} \%$ after HD. There was a significant interaction between age and change of HF\% $(p=0.023)$ after HD. After multivariate adjustments for clinical, biochemical data and medications, systolic blood pressure, total cholesterol, hemoglobin, and hemoglobin were associated with $\Delta \mathrm{LF}$, whereas cerebrovascular disease, systolic blood pressure, and fasting glucose were associated with $\Delta \mathrm{HF} \%$ in patients aged $\geq 65$ years.

Conclusion: Our study demonstrated significant changes in HRV after HD in diabetic patients. In the patients aged $\geq 65$ years, LF was increased, whereas HF\% was decreased significantly after HD. Among the HRV parameters, age had an interaction with the change of HF\%.

Keywords: heart rate variability, autonomic nervous system, age, diabetes, hemodialysis 


\section{INTRODUCTION}

Heart rate variability (HRV) is a non-invasive method of measuring variations in heart rate, and it can be used to evaluate cardiovascular (CV) autonomic neuropathy and activities of the autonomic nervous system (Camm et al., 1996). In practice, HRV is defined as variations in instantaneous heart rate and $\mathrm{R}-\mathrm{R}$ intervals measured using electrocardiography (ECG) (Camm et al., 1996). Abnormal HRV primarily reflects dysregulation between the sympathetic and parasympathetic nervous systems. Frequency-domain analysis of HRV has been increasingly used to assess the autonomic nervous system due to its non-invasiveness and availability.

A low HRV, indicating impaired autonomic function, has been reported in patients undergoing hemodialysis (HD), and it has been associated with adverse CV outcomes and even death in patients undergoing HD (Fukuta et al., 2003; Oikawa et al., 2009). Our recent study demonstrated that changes in HRV parameters before and after HD have a stronger predictive ability for overall and $\mathrm{CV}$ mortality than HRV parameters before HD (Chen et al., 2016). A previous study reported that HD patients with diabetes had a smaller degree of changes in HRV after HD than patients without diabetes, and that this may be related to diabetic autonomic neuropathy (Zitt et al., 2008). However, the factors associated with changes in HRV in diabetic HD patients have yet to be clarified.

Many neurological diseases of in the elderly such as dementia (Galimberti and Scarpini, 2016), Parkinson's disease (Pelosin et al., 2016) and cognitive impairment (Liu et al., 2017) have been reported to be caused by age-related cholinergic dysfunction. In addition to neurological diseases, cholinergic degeneration has also been reported to play a role in an obstructed bladder (Silva-Ramos et al., 2015) and constipation in the elderly, and especially in those with diabetes (Bharucha et al., 2013). The number of elderly patients undergoing HD has increased along with life expectancy, and these patients have been reported to be frailer (Fried et al., 2001) with greater functional and cognitive impairments compared to those who do not require HD (Anand et al., 2010). However, little is known about the association between age and autonomic regulation in $\mathrm{HD}$ patients with diabetes mellitus (DM). Therefore, the aims of this study were to investigate the relationship between age and HRV parameters before and after $\mathrm{HD}$, and further investigate the factors associated with change in HRV in diabetic patients.

\section{MATERIALS AND METHODS}

\section{Study Patients and Design}

This study was conducted at a regional hospital in southern Taiwan, and included all maintenance HD patients except for those receiving $\mathrm{HD}$ at night. A total of 84 diabetic patients (45 males and 39 females) were enrolled from May 2012 to July 2012. All of these patients underwent HD three times per week, with each session lasting for 3.5-4.5 h, a blood flow rate of $250-300 \mathrm{~mL} / \mathrm{min}$, and a dialysate flow of $500 \mathrm{~mL} / \mathrm{min}$. $K \mathrm{t} / \mathrm{V}$ was calculated from blood samples obtained before and after HD.

\section{Ethics Statement}

The study protocol was approved by the Institutional Review Board of Kaohsiung Medical University Hospital. Written informed consent was obtained from all of the enrolled patients, and the study was conducted according to the principles expressed in the Declaration of Helsinki. In addition, all of the patients consented to the inclusion and publication of their data in this study.

\section{Electrocardiogram Signal Processing}

Short-term power spectral analysis of HRV was performed in all of the enrolled patients in a quiet room with a temperature of $28^{\circ} \mathrm{C}$. The HRV analysis was performed according to standard protocols as reported previously (Kuo et al., 1999; Liu et al., 2003; Chen et al., 2009). Pericardial ECG was performed continuously for $5 \mathrm{~min}$ with the patients lying quietly in the supine position and breathing normally for at least $10 \mathrm{~min}$. The ECG examinations were performed $30 \mathrm{~min}$ before and $30 \mathrm{~min}$ after HD during the day ( 8 a.m. to 5 p.m.), and the signals were recorded using an HRV analyzer (SS1C, Enjoy Research, Taipei, Taiwan) which was equipped with an analog-to-digital converter and a sampling rate of $256 \mathrm{~Hz}$. The digitized ECG signals were acquired, stored, and processed using a computer. During analysis, each QRS complex was identified, and premature ventricular complexes and noise were removed using a standard QRS template using the software provided by the HRV analyzer. This was followed by resampling of the stationary $R-R$ values with interpolation at $7.11 \mathrm{~Hz}$ to provide consistency over a time period (Kuo et al., 1999).

\section{HRV Frequency-Domain Analysis}

Frequency-domain analysis was conducted using fast Fourier transformation. The direct current component was first removed, and then the effect of leakage was reduced using a Hamming window (Kuo and Chan, 1993). The power spectrum density was estimated based on FFT for each time segment (288 s; 2048 data points), and attenuation of the resulting power spectrum caused by sampling and the Hamming window was corrected. The power spectrum was then quantified into standard frequency-domain measurement including very low frequency (VLF) $(0.003-0.04 \mathrm{~Hz})$, high frequency (HF) $(0.15-0.40 \mathrm{~Hz})$, low frequency (LF) $(0.04-0.15 \mathrm{~Hz})$, total power $(0.003-0.40 \mathrm{~Hz})$ and $\mathrm{LF} / \mathrm{HF}$ ratio $\mathrm{HRV}$ as previously reported (Camm et al., 1996). HF was normalized to the percentage of total power to detect the sympathetic influence on HRV as follows: $\mathrm{HF} \%=\mathrm{HF} /\left(\right.$ total power-VLF) ${ }^{*} 100 \%$. All of the HRV parameters were logarithmically transformed to correct for skewness of the distribution (Camm et al., 1996).

\section{Collection of Demographic, Medical, and Laboratory Data}

Demographic and medical data were obtained from medical records and patient interviews, and included age, sex and co-morbidities. An autoanalyzer (COBAS Integra 400, Roche Diagnostics $\mathrm{GmbH}$, Mannheim, Germany) was used for all laboratory examinations which were conducted using fasting 
blood samples. The serum concentration of intact parathyroid hormone (iPTH) was measured using a commercially available two-sided immunoradiometric assay (CIS Bio International, Saclay, France). Ultrafiltration rate was defined as ultrafiltration divided by body weight. $\mathrm{Kt} / \mathrm{V}$ was used as a measure of dialysis efficiency, and was determined monthly using the Daugirdas method (Daugirdas, 1993).

\section{Statistical Analysis}

Statistical analysis was performed using SPSS for Windows version 15.0 (SPSS Inc. Chicago, IL, United States). HRV parameters are presented as percentage, mean \pm standard deviation, and mean \pm standard error of the mean. The duration of dialysis and levels of serum triglycerides and $\mathrm{PTH}$ are presented as median (25th-75th percentile). Differences between groups were analyzed using the chi-square test for categorical variables and the independent $t$-test for continuous variables with an approximately normal distribution, or the Mann-Whitney $U$ test for continuous variables with a skewed distribution. The Wilcoxon signed-rank test using nonparametric statistical analysis was carried out for comparisons of the HRV parameters before and after HD. Furthermore, two-ways repeated measures analysis of variance was used to test the interaction between age and change of HRV parameters after $\mathrm{HD}$. Changes in the HRV $(\triangle \mathrm{HRV})$ parameters were calculated as those measured after HD minus those measured before HD. Multiple stepwise linear regression analysis was used to identify the factors associated with $\triangle \mathrm{HRV}$ parameters. A $p$-value of less than 0.05 was considered to indicate a significant difference.

\section{RESULTS}

The mean age of the 84 enrolled patients with DM undergoing maintenance HD was $62.6 \pm 10.1$ years. Of these patients, $53.6 \%$ patients were male and $41.7 \%$ were $\geq 65$ years old. Comparisons of the baseline characteristics between the patients aged $<65$ years and $\geq 65$ years are shown in Table 1 . Compared to the patients younger than 65 years, those $\geq 65$ years of age were more predominantly female, and had lower systolic and diastolic blood pressures, lower total cholesterol, lower low-density lipoprotein (LDL) cholesterol and lower creatinine. Among all of the pre-dialysis HRV parameters, only HF\% was significantly higher in the patients $\geq 65$ years of age compared to those $<65$ years.

\section{Changes in HRV Parameters before and after HD}

In the patients aged $<65$ years, $\operatorname{VLF}(p=0.014), \operatorname{LF}(p<0.001)$, and HF $(p=0.030)$, were significantly increased after HD. In comparison, LF ( $p=0.043)$ was significantly increased in the patients $\geq 65$ years of age, but HF\% ( $p<0.007)$ was significantly decreased after HD (Table 2). Two-ways repeated measures analysis of variance was carried out to elucidate the interaction between age and change of HRV parameters after HD. Of note, the interaction between age and change of HF\% was significant $(p=0.023)$. There was no significant interaction between age and change of VLF $(p=0.852), \operatorname{LF}(p=0.316), \operatorname{HF}(p=0.189)$, and $\mathrm{LF} / \mathrm{HF}$ ratio $(p=0.117)$.

\section{Determinants of $\Delta \mathrm{HRV}$ Parameters in the Patients $<65$ Years Old}

Table 3 shows the unstandardized $\beta$ coefficients of $\triangle \mathrm{VLF}$, $\Delta \mathrm{LF}$, and $\Delta \mathrm{HF}$ which were significant in Table 2 in the patients <65 years old, after adjusting for age, sex, duration of $\mathrm{HD}$, a history of smoking, hypertension, coronary artery disease and cerebrovascular disease, systolic and diastolic blood pressure, albumin, fasting glucose, triglycerides, total cholesterol, LDL-cholesterol, hemoglobin, creatinine, potassium, total calcium, phosphorous, calcium-phosphorous product, $\mathrm{iPTH}$, uric acid, $K \mathrm{t} / \mathrm{V}$, ultrafiltration rate and medication. In the multivariate stepwise analysis, cerebrovascular disease $(\beta=-1.160, p=0.015)$, diastolic blood pressure $(\beta=-0.018$, $p=0.017)$, fasting glucose $(\beta=-0.016, p=0.001)$, hemoglobin $(\beta=-0.897, p=0.001)$, and beta-blocker use $(\beta=1.103$, $p=0.009)$ were independently associated with $\Delta$ VLF. Diastolic blood pressure $(\beta=-0.029, p=0.030)$, and ultrafiltration rate $(\beta=-0.272, p=0.033)$ were associated with $\Delta L F$. Cerebrovascular disease $(\beta=-0.765, p=0.049)$ was associated with $\triangle \mathrm{HF}$.

\section{Determinants of $\Delta \mathrm{HRV}$ Parameters in the Patients $\geq 65$ Years Old}

Table 4 presents the unstandardized $\beta$ coefficients of $\triangle \mathrm{LF}$ and $\triangle H F \%$ which were significant in Table 2 in the patients $\geq 65$ years old after adjusting for demographic, clinical and biochemical characteristics and medications. In the multivariate stepwise analysis, systolic blood pressure $(\beta=0.060, p=0.023)$, total cholesterol $(\beta=-0.146, p<0.001)$ and hemoglobin $(\beta=1.466, p<0.001)$ were associated with $\Delta$ LF. Furthermore, cerebrovascular disease $(\beta=12.762, p=0.047)$, systolic blood pressure $(\beta=0.357, p=0.012)$, and fasting glucose $(\beta=-0.126$, $p=0.003)$ were associated with $\Delta \mathrm{HF} \%$.

\section{DISCUSSION}

In the present study, we investigated the relationship between age and $\triangle \mathrm{HRV}$ in diabetic HD patients. We found that pre-dialytic $\mathrm{HF} \%$ was significantly higher in the patients $\geq 65$ years old than in those $<65$ years old. In addition, the patients $<65$ years old had increases in VLF, LF, and HF after HD, whereas the patients $\geq 65$ years old had an increase in LF and a decrease in $\mathrm{HF} \%$ after HD. Diastolic blood pressure, cerebrovascular disease, fasting glucose, hemoglobin, beta-blocker use and ultrafiltration rate were significantly associated with $\triangle \mathrm{HRV}$ in the patients $<65$ years old; whereas cerebrovascular disease, systolic blood pressure, fasting glucose, total cholesterol and hemoglobin were associated with $\triangle \mathrm{HRV}$ in the patients $\geq 65$ years old.

An increasing number of studies have investigated the effects of HD on the elderly (Anand et al., 2010). The first major finding of the current study is that VLF, LF, and HF increased 
TABLE 1 | Comparison of baseline characteristics between diabetes mellitus (DM) patients with age $<65$ and $\geq 65$ years old.

\begin{tabular}{|c|c|c|c|c|}
\hline Characteristics & All patients $(N=84)$ & Age $<65$ years $(N=49)$ & Age $\geq 65$ years $(N=35)$ & $p$ \\
\hline Male gender (\%) & 53.6 & 63.3 & 40.0 & 0.035 \\
\hline Smoking history (\%) & 27.4 & 32.7 & 20.0 & 0.200 \\
\hline Hypertension (\%) & 77.4 & 81.6 & 71.4 & 0.270 \\
\hline Coronary artery disease (\%) & 38.1 & 34.7 & 42.9 & 0.448 \\
\hline Systolic blood pressure (mmHg) & $164.0 \pm 26.0$ & $169.6 \pm 24.5$ & $155.6 \pm 26.2$ & 0.017 \\
\hline Diastolic blood pressure $(\mathrm{mmHg})$ & $84.1 \pm 14.8$ & $87.8 \pm 13.9$ & $78.6 \pm 14.6$ & 0.006 \\
\hline \multicolumn{5}{|l|}{ Laboratory parameters } \\
\hline Albumin (g/dL) & $3.8 \pm 0.3$ & $3.8 \pm 0.3$ & $3.7 \pm 0.3$ & 0.051 \\
\hline Fasting glucose (mg/dL) & $144.0 \pm 62.8$ & $133.4 \pm 47.2$ & $158.0 \pm 77.3$ & 0.102 \\
\hline Triglycerides (mg/dL) & $152.5(104-228.5)$ & $177(104.8-240.8)$ & $132.5(94.5-177.8)$ & 0.156 \\
\hline Potassium (mEq/L) & $4.5 \pm 0.7$ & $4.5 \pm 0.6$ & $4.4 \pm 0.7$ & 0.499 \\
\hline Total calcium (mg/dL) & $9.2 \pm 1.0$ & $9.3 \pm 0.9$ & $9.1 \pm 1.0$ & 0.439 \\
\hline Phosphorous (mg/dL) & $4.6 \pm 1.1$ & $4.6 \pm 1.1$ & $4.5 \pm 1.1$ & 0.634 \\
\hline Calcium-phosphorous product $\left(\mathrm{mg}^{2} / \mathrm{dL}^{2}\right)$ & $41.8 \pm 11.5$ & $42.8 \pm 11.6$ & $40.4 \pm 11.4$ & 0.359 \\
\hline iPTH (pg/mL) & $327.8(183.8-463.3)$ & $359.8(193.0-498.7)$ & 306.5 (177.9-364.9) & 0.525 \\
\hline Uric acid (mg/dL) & $7.6 \pm 1.9$ & $7.8 \pm 1.8$ & $7.3 \pm 2.0$ & 0.227 \\
\hline Kt/V (Daugirdes) & $1.5 \pm 0.3$ & $1.5 \pm 0.2$ & $1.6 \pm 0.4$ & 0.653 \\
\hline Ultrafiltration rate (\%) & $4.4 \pm 1.4$ & $4.3 \pm 1.3$ & $4.6 \pm 1.6$ & 0.415 \\
\hline \multicolumn{5}{|l|}{ Pre-dialysis HRV parameters } \\
\hline $\operatorname{VLF}\left(\mathrm{In} \mathrm{ms}^{2}\right)$ & $3.59 \pm 0.20$ & $3.38 \pm 0.21$ & $3.89 \pm 0.38$ & 0.215 \\
\hline $\mathrm{LF}\left(\ln \mathrm{ms}^{2}\right)$ & $1.39 \pm 0.48$ & $0.73 \pm 0.77$ & $2.32 \pm 0.38$ & 0.104 \\
\hline HF\% (nu) & $29.6 \pm 1.4$ & $30.4 \pm 1.8$ & $28.4 \pm 2.4$ & 0.516 \\
\hline LF/HF ratio & $0.23 \pm 0.13$ & $0.16 \pm 0.16$ & $0.34 \pm 0.22$ & 0.508 \\
\hline \multicolumn{5}{|l|}{ Medications } \\
\hline ACEl and/or ARB use & 28.6 & 28.2 & 29.2 & 0.935 \\
\hline Beta-blocker use & 30.2 & 25.6 & 37.5 & 0.319 \\
\hline Calcium channel blocker use & 31.7 & 28.2 & 37.5 & 0.441 \\
\hline
\end{tabular}

LDL, low-density lipoprotein; iPTH, intact parathyroid hormone; HRV, heart rate variability; $L F$, low frequency; $H F$, high frequency; $A C E I$, angiotensin converting enzyme inhibitor; ARB, angiotensin II receptor blocker.

significantly after $\mathrm{HD}$ in the patients aged $<65$ years, whereas LF increased during HD but HF\% decreased significantly in the patients $\geq 65$ years old. HF has been reported to represent respiratory sinus arrhythmia and vagal control of heart rate (Fouad et al., 1984). The function of autonomic nerve system of the elderly population might decline (Catai et al., 2014). Tan et al. (2017) reported a decrease in parasympathetic nervous activity during the nighttime postoperatively in elderly patients, and concluded that this may be due to higher sympathetic nervous activity resulting in a decrease in parasympathetic nervous activity. In addition, Genovesi et al. (2007) reported an increase in LF and a decrease in HF after HD, which is consistent with our findings and suggests impaired changes in the compensatory baroreflex-mediated activation of the sympathetic nervous system in elderly patients. Compared to pre-dialytic HRV, the changes of HRV during HD have been recognized as a stronger predictor of death, and impaired increase in HRV after HD was associated with mortality in HD patients (Chen et al., 2016). In our study, HRV increased significantly after $\mathrm{HD}$ in the patients aged $<65$ years, but HF\% was drastically decreased after HD in patients aged $\geq 65$ years. This finding implies that impaired parasympathetic 
TABLE 2 | Heart rate variability (HRV) parameters of DM patients with age $<65$ and $\geq 65$ years old before and after hemodialysis.

\begin{tabular}{|c|c|c|c|c|c|c|}
\hline \multirow[t]{2}{*}{ HRV parameters (frequency domain) } & \multicolumn{3}{|c|}{ Age $<65$ years } & \multicolumn{3}{|c|}{ Age $\geq 65$ years } \\
\hline & Before hemodialysis & After hemodialysis & $p$ & Before hemodialysis & After hemodialysis & $p$ \\
\hline VLF $\left(\ln m s^{2}\right)$ & $3.75(2.78-4.23)$ & $4.03(2.96-5.43)$ & 0.014 & $4.21(2.89-4.77)$ & $4.34(3.33-5.54)$ & 0.176 \\
\hline $\mathrm{LF}\left(\ln m s^{2}\right)$ & $1.52(0.69-2.44)$ & $2.34(1.41-3.81)$ & $<0.001$ & $2.55(1.36-3.26)$ & $3.43(1.53-4.63)$ & 0.043 \\
\hline $\mathrm{HF}\left(\ln \mathrm{ms}^{2}\right)$ & $1.89(0.94-2.78)$ & $2.31(1.50-3.11)$ & 0.030 & $2.98(1.88-3.85)$ & $2.53(1.74-3.90)$ & 0.905 \\
\hline HF\% (nu) & $31.2(20.1-43.1)$ & $29.8(22.2-37.8)$ & 0.885 & $39.5(29.4-51.0)$ & $28.5(18.1-39.0)$ & 0.007 \\
\hline LF/HF ratio & $-0.19(-1.11-0.77)$ & $0.11(-0.36-0.91)$ & 0.665 & $-0.14(-0.98-0.48)$ & $0.25(-0.52-1.17)$ & 0.091 \\
\hline
\end{tabular}

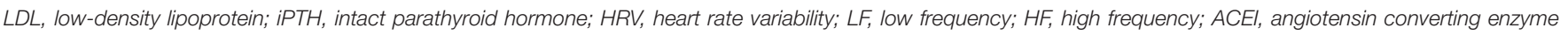
inhibitor; $A R B$, angiotensin II receptor blocker.

TABLE 3 | Determinants of $\triangle \mathrm{HRV}$ parameters of DM patients with age $<65$ years old.

\begin{tabular}{|c|c|c|}
\hline \multirow[t]{2}{*}{$\Delta \mathrm{HRV}$ parameters } & \multicolumn{2}{|l|}{ Multivariate (stepwise) } \\
\hline & $\begin{array}{c}\text { Unstandardized } \\
\text { coefficient } \beta(95 \% \mathrm{Cl})\end{array}$ & $p$ \\
\hline \multicolumn{3}{|l|}{$\Delta \mathrm{VLF}$} \\
\hline Cerebrovascular disease & $-1.160(-2.074,-0.245)$ & 0.015 \\
\hline Diastolic blood pressure (per $1 \mathrm{mmHg}$ ) & $-0.018(-0.032,-0.003)$ & 0.017 \\
\hline Fasting glucose (per 1 mg/dL) & $-0.016(-0.025,-0.007)$ & 0.001 \\
\hline Hemoglobin (per 1 g/dL) & $-0.897(-1.407,-0.387)$ & 0.001 \\
\hline Beta-blocker use & $1.103(0.299,1.906)$ & 0.009 \\
\hline \multicolumn{3}{|l|}{$\Delta \mathrm{LF}$} \\
\hline Diastolic blood pressure (per $1 \mathrm{mmHg}$ ) & $-0.029(-0.003,-0.054)$ & 0.030 \\
\hline Ultrafiltration rate (per $1 \%$ ) & $-0.272(-0.024,-0.520)$ & 0.033 \\
\hline \multicolumn{3}{|l|}{$\Delta \mathrm{HF}$} \\
\hline Cerebrovascular disease & $-0.765(-1.527,-0.003)$ & 0.049 \\
\hline \multicolumn{3}{|c|}{$\begin{array}{l}\text { Values expressed as unstandardized coefficient } \beta \text { and 95\% confidence interval (Cl). } \\
\text { Covariates in the multivariate model included age, sex, duration of dialysis, a history } \\
\text { of smoking, hypertension, coronary artery disease and cerebrovascular disease, } \\
\text { systolic and diastolic blood pressure, albumin, fasting glucose, triglycerides, total } \\
\text { cholesterol, LDL-cholesterol, hemoglobin, creatinine, potassium, total calcium, } \\
\text { phosphorous, calcium-phosphorous product, iPTH, uric acid, KtN } \text {, ultrafiltration } \\
\text { rate, and medications use including ACEI and/or ARB, Beta-blocker and calcium } \\
\text { channel blocker. }\end{array}$} \\
\hline
\end{tabular}

response to HD may be partly responsible for unfavorable outcomes in the elderly HD patients. Therefore, the present study appears that patients $\geq 65$ years old with DM had worse autonomic nervous system function after HD than patients $<65$ years old.

The current study also revealed that only pre-dialytic HF\% was significantly higher in the patients $\geq 65$ years old. Although HRV might be reduced with age, however, some studies showed the lack of negative correlation between age and HRV. Kalopita et al. (2014) found that HRV was not negatively correlated with age in diabetic patients. Furthermore, Amra et al. (2017) showed no correlation between age and HRV in diabetic group among patients with sleep apnea syndrome. McKune et al. (2017) even found that HF significantly increased with age in elderly people aged between 60 and 86 years, these findings are similar to the work by Reardon and Malik (1996) who were the first to examine HRV in the very elderly (age range $40-102$ years). They demonstrated that while global 24-h HRV decreased
TABLE 4 | Determinants of $\triangle H R V$ parameters of DM patients with age $\geq 65$ years old.

\begin{tabular}{lcc}
\hline \multirow{2}{*}{$\boldsymbol{H}$ HRV parameters } & \multicolumn{2}{c}{ Multivariate (stepwise) } \\
\cline { 2 - 3 } & $\begin{array}{c}\text { Unstandardized } \\
\text { coefficient } \boldsymbol{\beta}(\mathbf{9 5 \%} \mathbf{~ C l})\end{array}$ & $\boldsymbol{p}$ \\
\hline$\Delta \mathrm{LF}$ & & \\
Systolic blood pressure (per 1 mmHg) & $0.060(0.009,0.111)$ & 0.023 \\
Total cholesterol (per 1 mg/dL) & $-0.146(-0.173,-1.119)$ & $<0.001$ \\
Hemoglobin (per 1 g/dL) & $1.466(0.822,2.109)$ & $<0.001$ \\
$\Delta$ HF \% & & \\
Cerebrovascular disease & $12.762(0.216,25.309)$ & 0.047 \\
Systolic blood pressure (per 1 mmHg) & $0.357(0.090,0.625)$ & 0.012 \\
Fasting glucose (per 1 mg/dL) & $-0.126(-0.204,-0.048)$ & 0.003 \\
\hline
\end{tabular}

Covariates in the multivariate model included age, sex, duration of dialysis, a history of smoking, hypertension, coronary artery disease and cerebrovascular disease, systolic and diastolic blood pressure, albumin, fasting glucose, triglycerides, total cholesterol, LDL-cholesterol, hemoglobin, creatinine, potassium, total calcium, phosphorous, calcium-phosphorous product, iPTH, uric acid, Kt/V, ultrafiltration rate, and medications use including ACEI and/or ARB, Beta-blocker and calcium channel blocker.

with increasing age, HRV measures caused by parasympathetic modulation, such as pre-dialytic HF or HF\%, did not decline with age. The real cause remains to be studied in diabetic maintenance HD patients.

In the present study, ultrafiltration rate was associated with changes in LF after HD in the patients $<65$ years old. The process of ultrafiltration during HD reduces circulating volume and arterial pressure, which can be detected by baroreceptors to trigger a cardiac sympathetic response. Barnas et al. (1999) investigated autonomic nervous system changes during $\mathrm{HD}$, and reported that the LF component of HRV increased during nonhypotensive dialysis. Furthermore, systolic and diastolic blood pressures were associated with $\triangle \mathrm{HRV}$ in our diabetic patients undergoing HD. Dysregulation of the autonomic nervous system can lead to sustained hypertension, and subsequently to an increased risk of vascular complications (Palatini et al., 2006; Licht et al., 2013). Hypertension has also been associated with lower HRV (Schroeder et al., 2003), which suggests that compromised sympathetic-vagal balance in patients with hypertension. In addition, fluid status may also play a role in the association between blood pressure and changes in HRV. Ferrario et al. (2015) evaluated the effect of central volume on 
changes in the LF parameter of HRV in patients undergoing $\mathrm{HD}$, and found that patients with a low hydration status before HD had an increased LF after HD, whereas those with a high hydration status before HD exhibited no significant change in LF. A lower central volume caused by dialysis can lead to alterations in the capacity of the CV system and thereby changes in LF In patients with a low hydration status. However, removal of the same volume of fluid during HD may not reduce the central volume sufficiently to reactivate oscillations in frequency in patients with a high hydration status, and this may explain the associations between blood pressure, ultrafiltration rate and $\triangle \mathrm{HRV}$ in this study.

An increasing number of studies have reported an association between an imbalance of the autonomic nervous system and pathophysiological conditions, including, dyslipidemia, sudden death, coronary artery disease, heart failure, and CV risk factors. In the present study, total cholesterol was associated with reduction in $\mathrm{LF}$ after $\mathrm{HD}$ in the patients aged $\geq 65$ years old. A low HRV has been reported to able to predict intima media thickness (Dekker et al., 2000) and progression of coronary atherosclerosis (Huikuri et al., 1999) in patients with recent CV disorders. Manfrini et al. (2008) investigated whether hypercholesterolemic atherosclerosis leads to impairment of the vagus nerve and worsening of HRV (Manfrini et al., 2008), and found that an increase in plaque size and substantial arterial remodeling led to outward stretching of the vessel wall behind the plaque, and was associated with autonomic dysfunction due to impaired vagal tone. This may explain the positive association between total cholesterol and reduction in HRV after HD in patients with diabetes.

Hemoglobin level was positively correlated with change in LF after $\mathrm{HD}$ in patients $\geq 65$ years old in the present study. In line with our findings, a positive correlation between hemoglobin and LF was shown in HD patients ( $\mathrm{Ng}$ et al., 2017). This implied that better sympathetic compensation occur as a result of fluid removal during $\mathrm{HD}$ in patients with better hemoglobin level. Anemia might be related higher non-transferrin-bound iron and labile plasma iron levels, leading to oxidative stress-mediated cardiac autonomic dysfunction, thus resulting in reduced HRV (Wijarnpreecha et al., 2015).

A lower HRV has been reported in patients with hemorrhagic and ischemic stroke (Korpelainen et al., 1999; Colivicchi et al., 2004), and baroreflex impairment has also been reported in these patients (Sykora et al., 2008). In the current study, a history of cerebrovascular disease was associated with both decrease in VLF and HF after HD in the patients aged $<65$ years old, and decrease in $\mathrm{HF} \%$ after $\mathrm{HD}$ in the patients aged $\geq 65$ years old. In other words, cerebrovascular disease was associated with a smaller degree in the decrease in HF\% after HD. This implies that autonomic CV dysregulation and impaired parasympathetic reaction occur as a result of fluid removal during HD in patients with stroke.

Another finding of the current study is that fasting glucose was associated with a change in HRV in the diabetic patients. Zitt et al. (2008) investigated the effect of DM on blunted autonomic response in patients undergoing $\mathrm{HD}$, this suggests that impaired autonomic function may be associated with damage caused by diabetes in autonomic neuropathy. In the current study, we also found an association between a high level of fasting glucose and a smaller degree of change in $\mathrm{HF} \%$ after $\mathrm{HD}$, implying that patients with diabetes and a high level of fasting glucose may have an impaired sympathy-vagal equilibrium. Microangiopathy has also been reported to play an important role in the development of diabetic neuropathy (Wada et al., 1999, 2001). The advanced glycation end products interacts with their receptors, can have a biological effect on tissues resulting in the complications of diabetes, further contributing to endoneural vascular dysfunction, consequently peripheral nerve microangiopathy (Wada and Yagihashi, 2005) and peripheral neuropathy (Singh et al., 2014).

There are several limitations to the present study. First, HRV can be measured in frequency or time domain parameters; however, we only used the frequency domain HRV. Although frequency domain parameters have been reported to be well correlated ( $r=0.85$ ) with time domain parameters, several time domain parameters have been reported to be clinically relevant (Kleiger et al., 1987, 1991). Thus, time domain measurements need to be included in future studies. Second, spectral HRV measurements and their physiological interpretation are not clear as previously thought, and they also have many limitations. More recent measures of HRV (e.g., fractal/complexity/scale invariant measures, entropy-based measures) should also be investigated as spectral HRV measurements in future studies. Third, causal relationships in this cross-sectional study could not be confirmed, and future prospective studies are needed to address this issue. Fourth, the number of enrolled patients was relatively small, which may have limited the statistical power. The percentage of non-stationary segments was lacking. Finally, the circadian pattern of autonomic modulation of the heart rate has been reported to exhibit a reduced HRV during the day with increased sympathetic activity and increased HRV during the night with the predominance of vagal modulation (Molgaard et al., 1991; Chen et al., 2011). In the present study, in order to minimize the influence of the circadian rhythm, we performed all HRV examinations during the day (between 8 a.m. and 5 p.m.), however, the rhythm may have been lost in some patients and the influence could not be defined. A longer period of ECG recordings may have established a baseline for each patient.

\section{CONCLUSION}

Our results demonstrated significant changes in HRV after $\mathrm{HD}$ in diabetic patients. In the patients aged $\geq 65$ years, LF was increased, whereas $\mathrm{HF} \%$ was decreased significantly after HD. Among the HRV parameters, age had an interaction with the change of HF\%, suggesting impaired parasympathetic response to $\mathrm{HD}$ in the elderly patients. Moreover, systolic blood pressure, total cholesterol, hemoglobin, and hemoglobin were associated with $\Delta \mathrm{LF}$, whereas cerebrovascular disease, systolic blood pressure, and fasting glucose were associated with $\triangle \mathrm{HF} \%$ in patients aged $\geq 65$ years. Further prospective studies and follow-up are needed to validate the effects of aging and HRV on the outcomes of patients with diabetes. 


\section{AUTHOR CONTRIBUTIONS}

Conceived and designed the experiments and analyzed the data: S-WN, J-CH, and S-CC. Performed the experiments: S-WN, $\mathrm{J}-\mathrm{CH}, \mathrm{HL}$, and I-CK. Contributed reagents/materials/analysis tools: J-CH, P-YW, S-CC, Y-WC, and J-MC. Wrote the manuscript: $\mathrm{S}-\mathrm{WN}$ and J-CH.

\section{REFERENCES}

Amra, B., Behjati, M., Penzel, T., Fietze, I., Schoebel, C., and Sarrafzadegan, N. (2017). Nocturnal heart rate variation in diabetic and non-diabetic patients with sleep apnea syndrome. Sleep Med. 29, 57-60. doi: 10.1016/j.sleep.2016.11.003

Anand, S., Kurella Tamura, M., and Chertow, G. M. (2010). The elderly patients on hemodialysis. Minerva Urol. Nefrol. 62, 87-101.

Barnas, M. G., Boer, W. H., and Koomans, H. A. (1999). Hemodynamic patterns and spectral analysis of heart rate variability during dialysis hypotension. J. Am. Soc. Nephrol. 10, 2577-2584.

Bharucha, A. E., Low, P., Camilleri, M., Veil, E., Burton, D., Kudva, Y., et al. (2013). A randomised controlled study of the effect of cholinesterase inhibition on colon function in patients with diabetes mellitus and constipation. Gut 62 , 708-715. doi: 10.1136/gutjnl-2012-302483

Camm, A. J., Malik, M., Bigger, J., Breithardt, G., Cerutti, S., Cohen, R., et al. (1996). Heart rate variability: standards of measurement, physiological interpretation and clinical use. Task force of the European society of cardiology and the North American society of pacing and electrophysiology. Circulation 93, 1043-1065. doi: 10.1161/01.CIR.93.5.1043

Catai, A. M., Takahashi, A., Perseguini, N. M., Milan, J. C., Minatel, V., RehderSantos, P., et al. (2014). Effect of the postural challenge on the dependence of the cardiovascular control complexity on age. Entropy 16, 6686-6704. doi: $10.3390 / \mathrm{e} 16126686$

Chen, C. J., Kuo, T. B., Tseng, Y. J., and Yang, C. C. (2009). Combined cardiac sympathetic excitation and vagal impairment in patients with non-organic erectile dysfunction. Clin. Neurophysiol. 120, 348-352. doi: 10.1016/j.clinph. 2008.10.155

Chen, S. C., Huang, J. C., Tsai, Y. C., Hsiu-Chin Mai, R. N., Jui-Hsin Chen, R. N., Kuo, P. L., et al. (2016). Heart rate variability change before and after hemodialysis is associated with overall and cardiovascular mortality in hemodialysis. Sci. Rep. 6:20597. doi: 10.1038/srep20597

Chen, S. C., Su, H. M., Hung, C. C., Chang, J. M., Liu, W. C., Tsai, J. C., et al. (2011). Echocardiographic parameters are independently associated with rate of renal function decline and progression to dialysis in patients with chronic kidney disease. Clin. J. Am. Soc. Nephrol. 6, 2750-2758. doi: 10.2215/cjn.04660511

Colivicchi, F., Bassi, A., Santini, M., and Caltagirone, C. (2004). Cardiac autonomic derangement and arrhythmias in right-sided stroke with insular involvement. Stroke 35, 2094-2098. doi: 10.1161/01.STR.0000138452.81003.4c

Daugirdas, J. T. (1993). Second generation logarithmic estimates of single-pool variable volume Kt/V: an analysis of error. J. Am. Soc. Nephrol. 4, 1205-1213.

Dekker, J. M., Crow, R. S., Folsom, A. R., Hannan, P. J., Liao, D., Swenne, C. A., et al. (2000). Low heart rate variability in a 2-minute rhythm strip predicts risk of coronary heart disease and mortality from several causes: the ARIC study. Atherosclerosis risk in communities. Circulation 102, 1239-1244. doi: 10.1161/01.CIR.102.11.1239

Ferrario, M., Moissl, U., Garzotto, F., Cruz, D. N., Tetta, C., Signorini, M. G., et al. (2015). The forgotten role of central volume in low frequency oscillations of heart rate variability. PLoS One 10:e0120167. doi: 10.1371/journal.pone. 0120167

Fouad, F. M., Tarazi, R. C., Ferrario, C. M., Fighaly, S., and Alicandri, C. (1984). Assessment of parasympathetic control of heart rate by a noninvasive method. Am. J. Physiol. 246, H838-H842. doi: 10.1152/ajpheart.1984.246.6.H838

Fried, L. P., Tangen, C. M., Walston, J., Newman, A. B., Hirsch, C., Gottdiener, J., et al. (2001). Frailty in older adults: evidence for a phenotype. J. Gerontol. A Biol. Sci. Med. Sci. 56, M146-M156. doi: 10.1093/gerona/56.3.M146

Fukuta, H., Hayano, J., Ishihara, S., Sakata, S., Mukai, S., Ohte, N., et al. (2003). Prognostic value of heart rate variability in patients with end-stage renal disease

\section{ACKNOWLEDGMENTS}

The authors wish to thank Statistical Analysis Laboratory, Department of Medical Research, Kaohsiung Medical University Hospital and Kaohsiung Municipal HsiaoKang Hospital, Kaohsiung Medical University for their assistance.

on chronic haemodialysis. Nephrol. Dial. Transplant. 18, 318-325. doi: 10.1093/ ndt/18.2.318

Galimberti, D., and Scarpini, E. (2016). Old and new acetylcholinesterase inhibitors for Alzheimer's disease. Expert Opin. Investig. Drugs 25, 1181-1187. doi: $10.1080 / 13543784.2016 .1216972$

Genovesi, S., Bracchi, O., Fabbrini, P., Luisetto, E., Vigano, M. R., Lucini, D., et al. (2007). Differences in heart rate variability during haemodialysis and haemofiltration. Nephrol. Dial. Transplant. 22, 2256-2262. doi: 10.1093/ndt/ gfm 125

Huikuri, H. V., Jokinen, V., Syvanne, M., Nieminen, M. S., Airaksinen, K. E., Ikaheimo, M. J., et al. (1999). Heart rate variability and progression of coronary atherosclerosis. Arterioscler. Thromb. Vasc. Biol. 19, 1979-1985. doi: 10.1161/ 01.ATV.19.8.1979

Kalopita, S., Liatis, S., Thomakos, P., Vlahodimitris, I., Stathi, C., Katsilambros, N., et al. (2014). Relationship between autonomic nervous system function and continuous interstitial glucose measurement in patients with type 2 diabetes. J. Diabetes. Res. 2014:835392. doi: 10.1155/2014/835392

Kleiger, R. E., Bigger, J. T., Bosner, M. S., Chung, M. K., Cook, J. R., Rolnitzky, L. M., et al. (1991). Stability over time of variables measuring heart rate variability in normal subjects. Am. J. Cardiol. 68, 626-630. doi: 10.1016/0002-9149(91) 90355-O

Kleiger, R. E., Miller, J. P., Bigger, J. T. Jr., and Moss, A. J. (1987). Decreased heart rate variability and its association with increased mortality after acute myocardial infarction. Am. J. Cardiol. 59, 256-262. doi: 10.1016/0002-9149(87) 90795-8

Korpelainen, J. T., Sotaniemi, K. A., Makikallio, A., Huikuri, H. V., and Myllyla, V. V. (1999). Dynamic behavior of heart rate in ischemic stroke. Stroke 30, 1008-1013. doi: 10.1161/01.STR.30.5.1008

Kuo, T. B., and Chan, S. H. (1993). Continuous, on-line, real-time spectral analysis of systemic arterial pressure signals. Am. J. Physiol. 264, H2208-H2213. doi: 10.1152/ajpheart.1993.264.6.H2208

Kuo, T. B., Lin, T., Yang, C. C., Li, C. L., Chen, C. F., and Chou, P. (1999). Effect of aging on gender differences in neural control of heart rate. Am. J. Physiol. 277, H2233-H2239. doi: 10.1152/ajpheart.1999.277.6.H2233

Licht, C. M., de Geus, E. J., and Penninx, B. W. (2013). Dysregulation of the autonomic nervous system predicts the development of the metabolic syndrome. J. Clin. Endocrinol. Metab. 98, 2484-2493. doi: 10.1210/jc.20123104

Liu, C. C., Kuo, T. B., and Yang, C. C. (2003). Effects of estrogen on genderrelated autonomic differences in humans. Am. J. Physiol. Heart Circ. Physiol. 285, H2188-H2193. doi: 10.1152/ajpheart.00256.2003

Liu, Q., Zhu, Z., Teipel, S. J., Yang, J., Xing, Y., Tang, Y., et al. (2017). White matter damage in the cholinergic system contributes to cognitive impairment in subcortical vascular cognitive impairment, no dementia. Front. Aging Neurosci. 9:47. doi: 10.3389/fnagi.2017.00047

Manfrini, O., Pizzi, C., Viecca, M., and Bugiardini, R. (2008). Abnormalities of cardiac autonomic nervous activity correlate with expansive coronary artery remodeling. Atherosclerosis 197, 183-189. doi: 10.1016/j.atherosclerosis.2007. 03.013

McKune, A. J., Peters, B., Ramklass, S. S., van Heerden, J., Roberts, C. Krejci, J., et al. (2017). Autonomic cardiac regulation, blood pressure and cardiorespiratory fitness responses to different training doses over a 12 week group program in the elderly. Arch. Gerontol. Geriatr. 70, 130-135. doi: 10.1016/ j.archger.2017.01.012

Molgaard, H., Sorensen, K. E., and Bjerregaard, P. (1991). Circadian variation and influence of risk factors on heart rate variability in healthy subjects. Am. J. Cardiol. 68, 777-784. doi: 10.1016/0002-9149(91)90653-3 
Ng, H. Y., Hsueh, S. K., Lee, Y. T., Chiou, T. T., Huang, P. C., and Lee, C. T. (2017). Synergic impact of vascular calcification and low autonomic tone in mortality of hemodialysis patients. Nephron 137, 91-98. doi: 10.1159/00047 7827

Oikawa, K., Ishihara, R., Maeda, T., Yamaguchi, K., Koike, A., Kawaguchi, H., et al. (2009). Prognostic value of heart rate variability in patients with renal failure on hemodialysis. Int. J. Cardiol. 131, 370-377. doi: 10.1016/j.ijcard.2007. 10.033

Palatini, P., Longo, D., Zaetta, V., Perkovic, D., Garbelotto, R., and Pessina, A. C. (2006). Evolution of blood pressure and cholesterol in stage 1 hypertension: role of autonomic nervous system activity. J. Hypertens. 24, 1375-1381. doi: 10.1097/01.hjh.0000234118.25401.1c

Pelosin, E., Ogliastro, C., Lagravinese, G., Bonassi, G., Mirelman, A., Hausdorff, J. M., et al. (2016). Attentional control of gait and falls: is cholinergic dysfunction a common substrate in the elderly and Parkinson's disease? Front. Aging Neurosci. 8:104. doi: 10.3389/fnagi.2016.00104

Reardon, M., and Malik, M. (1996). Changes in heart rate variability with age. Pacing Clin. Electrophysiol. 19, 1863-1866. doi: 10.1111/j.1540-8159.1996. tb03241.x

Schroeder, E. B., Liao, D., Chambless, L. E., Prineas, R. J., Evans, G. W., and Heiss, G. (2003). Hypertension, blood pressure, and heart rate variability: the Atherosclerosis risk in communities (ARIC) study. Hypertension 42, 1106-1111. doi: 10.1161/01.hyp.0000100444.71069.73

Silva-Ramos, M., Silva, I., Faria, M., Magalhaes-Cardoso, M. T., Correia, J., Ferreirinha, F., et al. (2015). Impairment of ATP hydrolysis decreases adenosine A1 receptor tonus favoring cholinergic nerve hyperactivity in the obstructed human urinary bladder. Purinergic Signal. 11, 595-606. doi: 10.1007/s11302015-9478-z

Singh, V. P., Bali, A., Singh, N., and Jaggi, A. S. (2014). Advanced glycation end products and diabetic complications. Korean J. Physiol. Pharmacol. 18, 1-14. doi: 10.4196/kjpp.2014.18.1.1

Sykora, M., Diedler, J., Rupp, A., Turcani, P., Rocco, A., and Steiner, T. (2008). Impaired baroreflex sensitivity predicts outcome of acute intracerebral hemorrhage. Crit. Care. Med. 36, 3074-3079. doi: 10.1097/CCM.0b013e3 $1818 \mathrm{~b} 306 \mathrm{~d}$
Tan, C., Saito, N., and Miyawaki, I. (2017). Changes in heart rate and autonomic nervous activity after orthopedic surgery in elderly Japanese patients. Kobe J. Med. Sci. 62, E129-E135.

Wada, R., Nishizawa, Y., Yagihashi, N., Takeuchi, M., Ishikawa, Y., Yasumura, K., et al. (2001). Effects of OPB-9195, anti-glycation agent, on experimental diabetic neuropathy. Eur. J. Clin. Invest. 31, 513-520. doi: 10.1046/j.1365-2362.2001. 00826.x

Wada, R., Sugo, M., Nakano, M., and Yagihashi, S. (1999). Only limited effects of aminoguanidine treatment on peripheral nerve function, $(\mathrm{Na}+, \mathrm{K}+)$-ATPase activity and thrombomodulin expression in streptozotocin-induced diabetic rats. Diabetologia 42, 743-747. doi: 10.1007/s001250051223

Wada, R., and Yagihashi, S. (2005). Role of advanced glycation end products and their receptors in development of diabetic neuropathy. Ann. N. Y. Acad. Sci. 1043, 598-604. doi: 10.1196/annals.1338.067

Wijarnpreecha, K., Siri-Angkul, N., Shinlapawittayatorn, K., Charoenkwan, P., Silvilairat, S., Siwasomboon, C., et al. (2015). Heart rate variability as an alternative indicator for identifying cardiac iron status in non-transfusion dependent Thalassemia patients. PLoS One 10:e0130837. doi: 10.1371/journal. pone. 0130837

Zitt, E., Neyer, U., Meusburger, E., Tiefenthaler, M., Kotanko, P., Mayer, G., et al. (2008). Effect of dialysate temperature and diabetes on autonomic cardiovascular regulation during hemodialysis. Kidney. Blood. Press. Res. 31, 217-225. doi: 10.1159/000141926

Conflict of Interest Statement: The authors declare that the research was conducted in the absence of any commercial or financial relationships that could be construed as a potential conflict of interest.

Copyright (C) 2018 Niu, Huang, Chen, Lin, Kuo, Wu, Chiu and Chang. This is an open-access article distributed under the terms of the Creative Commons Attribution License (CC BY). The use, distribution or reproduction in other forums is permitted, provided the original author(s) and the copyright owner are credited and that the original publication in this journal is cited, in accordance with accepted academic practice. No use, distribution or reproduction is permitted which does not comply with these terms. 